TRABAJO Y TRABAJADORES:

RED LATINOAMERICANA

ENSAYO BIBLIOGRÁFICO

\title{
Branko Milanovic y la desigualdad económica en perspectiva histórica: un diálogo posible con la historia del trabajo
}

\author{
LUCAS POY
}

Consejo Nacional de Investigaciones Científicas y Técnicas - Centro de Estudios Históricos de los Trabajadores y las Izquierdas, Argentina Instituto Internacional de Historia Social, Países Bajos lucaspoy@gmail.com

Trabajos reseñados:

Branko Milanovic, Global Inequality. A New Approach for the Age of Globalization (Cambridge: Harvard University Press, 2016).

Branko Milanovic, Capitalism, Alone. The Future of the System that Rules the World (Cambridge, Harvard University Press, 2019). 
Las múltiples desigualdades que atraviesan las sociedades humanas han sido siempre un tema de interés tanto desde una perspectiva analítica como política. ${ }^{1}$ En particular, la pregunta por las diferencias en cuanto al acceso a recursos materiales -la llamada desigualdad vertical- ocupa un lugar destacado en las inquietudes de numerosos científicos sociales, historiadores y economistas y ha ganado trascendencia en los últimos años, en particular después de la popularidad alcanzada por El capital en el siglo XXI, el libro de Thomas Piketty publicado en 2013.2 Es evidente que la recurrencia del tema es consecuencia de la crisis económica de fines de la década de 2000 y del sostenido incremento de la desigualdad de ingresos que se observa en muchos países en las últimas décadas. Una expresión de ello es la utilización de términos referenciados en la estadística por parte de movimientos sociales que denuncian al “1\%” y se reivindican expresión del “99\%”.

Si bien Piketty es el más conocido, son varios los autores que han recibido atención en este último tiempo por sus estudios sobre desigualdad económica. Uno de ellos es el serbio-estadounidense Branko Milanovic, nacido en 1953 en la ex Yugoslavia, quien se desempeñó durante mucho tiempo como economista jefe del departamento de investigación del Banco Mundial y actualmente trabaja en la City University of New York. Si bien los trabajos de Milanovic muestran una fuerte tendencia a extenderse en recomendaciones y sugerencias a gobiernos y empresas al igual que los de Piketty, también desarrollan interpretaciones de carácter histórico; esto último es lo que motiva las reflexiones que siguen a continuación. A diferencia de lo que ocurre en El capital en el siglo XXI, las obras de Milanovic examinan la evolución de la desigualdad con una escala de análisis que no se limita a Europa y Estados Unidos sino que propone una perspectiva global. ${ }^{3}$ Estas notas tienen un objetivo doble: por un lado, procuran presentar al público interesado en el tema un resumen de los principales aportes y argumentos de los últimos dos libros publicados por Milanovic acerca de la desigualdad material en perspectiva histórica; por el otro, introducen algunas

1 Este trabajo fue elaborado en el marco del Proyecto PUE-CONICET "Las dimensiones de la desigualdad en la larga duración. Economía, sociedad y política en el espacio rioplatense, siglos XVI a XX” (Instituto Ravignani). Me resultaron muy importantes los comentarios y críticas de Hernán Camarero, Martín Cuesta y Santiago Poy.

2 La versión original, con el título Le Capital au XXIe siècle, fue publicada en francés por Seuil en 2013. La versión en español fue editada por Fondo de Cultura Económica en 2014.

3 Algo que Piketty ha reconocido y tratado de subsanar en su nuevo libro Capital e ideología (Barcelona: Planeta, 2019). Ver, en particular, la "Advertencia al lector y agradecimientos". 
breves reflexiones acerca de los aportes que, en mi opinión, puede hacer la historia del trabajo a los debates en curso.4

Un eje que atraviesa los argumentos de Milanovic en los dos volúmenes que examinamos aquí es una crítica, y al mismo tiempo una propuesta de reformulación, a la llamada "curva de Kuznets". Hace referencia a la tesis, elaborada en la década de 1950 por el economista Simon Kuznets (19011985), según la cual la desigualdad en una sociedad es muy baja cuando los niveles de ingreso son muy reducidos, luego crece cuando se desarrolla la economía y finalmente vuelve a reducirse cuando se alcanzan niveles de ingreso más elevados. Milanovic considera que esta hipótesis ha quedado muy cuestionada dado que, si bien es cierto que creció fuertemente desde el despegue industrial y luego se redujo a partir de 1914, la desigualdad en los países desarrollados ha vuelto a dispararse en las últimas décadas.

Para corregir los problemas que encuentra en la hipótesis, y evitar al mismo tiempo desechar por completo los aportes de Kuznets, Milanovic sostiene que la "curva" debe en realidad reformularse y entender como una sucesión de "olas o ciclos". La idea, que el autor presenta en Global Inequality pero que atraviesa también las argumentaciones de su obra más reciente, sostiene que, si ampliamos la mirada históricamente, no habría una única curva sino una sucesión de etapas de ascenso y descenso de la desigualdad que se repiten a lo largo del tiempo. Milanovic sostiene también que estos ciclos se presentan de manera diferente en las etapas preindustriales respecto a la época capitalista y que es preciso analizar los acontecimientos del siglo XX a escala global para comprender las peculiaridades de la desigualdad económica mundial en nuestros días. El planteo tiene largos alcances y aborda un período de tiempo prolongado, por lo cual resulta de especial interés para el análisis histórico.

\section{La desigualdad antes y después de la Revolución Industrial}

Según Milanovic, es posible advertir ciclos de aumento y descenso de la desigualdad a lo largo de extensos períodos históricos. El autor introduce, sin embargo, una distinción importante: antes de la Revolución Industrial estos ciclos operaban en sociedades con economías estancadas, donde se

4. Buena parte de las argumentaciones presentadas por Milanovic en estos libros ya habían sido avanzadas en dos obras previas: Worlds Apart. Measuring International and Global Inequality (Princeton/Oxford: Princeton University Press, 2005) y The Haves and the Have-Nots: A Brief and Idiosyncratic History of Global Inequality (Nueva York: Basic Books 2010). 
mantenía estable lo que llama el "ingreso mediano" y no era posible advertir, por lo tanto, una relación entre nivel de ingreso y nivel de desigualdad. En las sociedades preindustriales de estas características, sostiene Milanovic, el enriquecimiento y la prosperidad de las clases ricas llevaba necesariamente a un incremento de la desigualdad y que por lo tanto solo podía reducirse por causas "malignas" de tipo catastrófico, como guerras y epidemias, que destruían riqueza y también diezmaban a la población. Luego, el ciclo podía volver a comenzar, en un juego de suma cero en términos de crecimiento económico.

Dado que no realizó investigaciones propias sobre estos temas, Milanovic se apoya en otros investigadores para respaldar estos argumentos en lo referido al período preindustrial. En particular, utiliza un trabajo de Carlos Álvarez Nogal y Leandro Prados de la Escosura que analiza el caso español a través de un período de más de cinco siglos y advierte marcados ciclos de ascenso y descenso de la desigualdad con un nivel de ingresos que se mantenía estancado. Según estos autores, las dos grandes fuerzas que redujeron la desigualdad fueron la peste del siglo XIV, las guerras a partir de la segunda mitad del siglo XVI y las guerras napoleónicas a comienzos del siglo XIX. Milanovic también toma conclusiones de un trabajo similar de Guido Alfani para ciudades del norte de Italia, que encuentra una reducción significativa de la desigualdad como consecuencia de la peste del siglo XIV.

Las inquietudes de Milanovic, en cualquier caso, tienen como eje del análisis la sociedad capitalista. Es por eso que subraya el cambio sustancial que se produjo con el advenimiento de la revolución industrial, cuando el nivel de ingreso comenzó a crecer aceleradamente y permitió un incremento notable de la desigualdad material. Si bien el autor tampoco examina en detalle la transición al capitalismo y el proceso de industrialización, mucho menos con investigaciones propias, es evidente que se refiere a un hecho fácil de constatar: el fenomenal salto tecnológico y productivo que acompañó al desarrollo capitalista en Europa y Estados Unidos permitió que todos los sectores de esas sociedades aumentasen su ingreso en términos absolutos; obviamente, dado que unos se enriquecieron mucho más que otros, la desigualdad aumentó en forma significativa. Estamos aquí en la parte ascendente de la "curva de Kuznets" tradicional.

Al mismo tiempo -y este es posiblemente uno de los principales aportes de Milanovic al debate contemporáneo-, el autor recuerda que con el despegue industrial de los países ricos se produjo una divergencia sustantiva entre regiones del planeta porque la desigualdad entre Europa occidental y Norteamérica y el resto del mundo no industrializado se hizo mucho más 
significativa. No solamente aumentó la desigualdad económica al interior de cada país (industrializado) sino también la desigualdad material entre países. Según Milanovic, a principios del siglo XIX, es decir antes de esta gran divergencia, lo más importante para determinar la desigualdad de ingresos era el lugar que ocupara una persona en la estructura de clases. La desigualdad global se explicaba sobre todo por las diferencias que existían entre ricos y pobres en todas las regiones, y no tanto por la diferencia entre países. Esto cambió completamente a partir del despegue industrial de los países capitalistas avanzados: la desigualdad económica global pasó a depender muchísimo más del lugar del mundo en el que una persona hubiera nacido, y no tanto de la clase a la que pertenecía (dicho de otro modo, los trabajadores europeos o norteamericanos reciben ingresos superiores a los ricos de un país pobre). En los términos de Milanovic, la revolución industrial "es similar a un Big Bang que puso a una parte de la humanidad en un camino de mayores ingresos y crecimiento sostenido, mientras que la mayoría se quedó donde estaba, y una parte incluso retrocedió". ${ }^{5}$

\section{El “siglo XX corto” y la reducción de la desigualdad económica}

El mundo se transformó decisivamente con el estallido de la Primera Guerra Mundial y el inicio de lo que Eric Hobsbawm llamó, de manera ya célebre, el "siglo XX corto". El corte cronológico resulta pertinente también en el terreno del debate aquí analizado, dado que es posible constatar, a lo largo de este "siglo de catástrofes", una significativa reducción de la desigualdad de ingresos en los países avanzados: lo que Kuznets graficó en la parte descendente (y definitiva, en su perspectiva) de su curva. Basándose en abundantes estadísticas elaboradas a partir de los ingresos de las unidades domésticas -disponibles en el sitio web del autor-, Milanovic aborda el problema, brinda una cantidad de datos que cuantifican este proceso de reducción de la desigualdad económica en términos de relación entre coeficiente Gini e ingreso, y advierte que existen dos tipos de explicaciones para el fenómeno. La que llama tradicional, expresada por el propio Kuznets, sostenía que la reducción de la desigualdad de ingresos fue producto de "fuerzas económicas": el aumento de la escolarización, el cierre de la etapa de transformaciones estructurales que llevaba población del campo a la ciudad y por lo tanto reforzaba la desigualdad entre la población urbana y la rural, el

5 Branko Milanovic, Global Inequality. A New Approach for the Age of Globalization. (Cambridge: Harvard University Press, 2016), 119. Las traducciones al español son mías. 
envejecimiento de la población y la demanda por seguridad social, etcétera. Desde esta perspectiva, el desarrollo económico (capitalista) tiene un carácter inherentemente virtuoso, que por su tendencia al crecimiento tiende a reducir las desigualdades de ingresos una vez llegado a cierto punto de su desarrollo; no es casual que la "curva de Kuznets" fuera un insumo clave de la llamada "teoría del derrame” en clave neoliberal.

La segunda explicación, por contraste, sostiene que la reducción de la desigualdad económica fue en realidad una consecuencia del impacto de las guerras, la acción de los sindicatos y de los partidos socialistas y comunistas. Se trata del argumento fundamental de Thomas Piketty: la reducción de la desigualdad del siglo XX en los países desarrollados habría sido un fenómeno excepcional, antes que una tendencia intrínseca del capitalismo en su desarrollo, como creía Kuznets. La tendencia fundamental del capitalismo es el aumento de la desigualdad, sostiene Piketty en su tesis, porque la tasa de incremento de los retornos del capital siempre supera a la tasa de incremento de los ingresos salariales.

La interpretación de Milanovic tiende a coincidir con Piketty en este aspecto central, pero cuestiona que estos factores políticos aparezcan -en el razonamiento del renombrado economista francés- como algo fundamentalmente "exógeno". Milanovic señala que los mismos deben ser integrados en la propia lógica de funcionamiento del sistema. Para hacerlo, plantea un argumento que parece bastante elemental para especialistas de este período y campo temático: sostiene que las guerras de la primera mitad del siglo XX no fueron un elemento "exógeno" sino una consecuencia de las desigualdades preexistentes. El propio autor reconoce que en este punto está yendo hacia una interpretación "más antigua y más convincente": la que considera que la guerra fue causada por la competencia imperialista, que a su vez tiene su origen en las desigualdades en la distribución del ingreso a nivel nacional.

Su conclusión, en cualquier caso, es que "las fuerzas malignas que quebraron el primer ciclo de Kuznets y establecieron una tendencia a la baja de la desigualdad en los países ricos por los siguientes 70 años estaban contenidas en la elevada e insostenible desigualdad doméstica que existía anteriormente". ${ }^{6}$ Fue la acción del movimiento obrero a fines del siglo XIX y comienzos del siglo XX, a pesar de sus derrotas, lo que "ejerció una presión que en última instancia resultó demasiado fuerte y dio como resultado una reducción sostenida en la desigualdad durante lo que suele llamarse el siglo

$6 \quad$ Ibídem, 96. 
XX corto". Las "políticas favorables a los ricos" se habrían visto limitadas por "la fuerza de los sindicatos, el poder político de los partidos socialistas y comunistas y el ejemplo y la amenaza militar de la Unión Soviética”. ${ }^{7}$

\section{Después de Reagan y Thatcher: aumento de la desigualdad de ingresos en los países ricos y disminución a nivel global}

A lo largo del "siglo XX corto", entonces, la desigualdad económica tendió a reducirse en los países más desarrollados, pero el hecho de que sus economías siguieran creciendo hizo que la distancia con los países más pobres se incrementase significativamente; como dijimos, la clave de la tesis de Milanovic es esta distinción entre desigualdad "intra" y desigualdad "entre". El punto máximo de la desigualdad global, según Milanovic, se ubica en algún momento entre 1970 y 1990. A partir de entonces se pusieron en movimiento dos tendencias contrapuestas pero unidas por un elemento común: la globalización de la economía internacional, inseparable de la caída de los regímenes comunistas en la Unión Soviética y Europa oriental, y la transición al capitalismo en China. Por un lado, recuerda Milanovic, todas las observaciones coinciden en que se incrementó la desigualdad de ingresos en los países ricos, algo que supuso el fin de la tendencia a la reducción de la desigualdad que había caracterizado al "siglo XX corto" y, por lo tanto, invalida la tendencia de la "curva de Kuznets". Por otro lado, la globalización implicó al mismo tiempo un fenomenal crecimiento de las economías asiáticas (fundamentalmente China), lo cual permitió que un amplio sector de su población, que partía de niveles de ingresos extremadamente bajos, mejorara su situación. Dado el enorme peso demográfico de las economías asiáticas, esto dio como resultado, según Milanovic, una reducción de la desigualdad económica a nivel global. Entre la década de 1950 y la de 1970, el PBI per cápita de Estados Unidos era 20 veces superior al de China. En 2010 esa proporción se había reducido a 4 a 1 :

La revolución económica en China fue seguida por aceleraciones del crecimiento similares en India, Vietnam, Tailandia, Indonesia y otros lugares de Asia. Aunque este crecimiento se vio acompañado por un incremento de la desigualdad dentro de cada uno de los países (especialmente en China), el cierre de la brecha con Occidente ayudó a reducir la desigualdad de ingresos a nivel global. Esto es lo que explica la reciente caída en el Gini global. ${ }^{8}$

7 Ibídem, 86-87.

8 Ibídem, 8. 
En Global Inequality, Milanovic resume sus hallazgos sobre la desigualdad global en las últimas tres décadas identificando tres tendencias significativas en el período que va desde 1988 hasta 2008. En primer lugar, el ascenso de lo que llama la "clase media global" (sectores de la población china y otros países asiáticos que mejoraron significativamente su nivel de ingresos). Milanovic muestra, por ejemplo, que el ingreso real per cápita de los dos quintiles medios en China se multiplicó por 3 (zonas urbanas) y por 2,2 (en zonas rurales), entre 1988 y 2008, y que se observan cifras similares en Indonesia, Vietnam y Tailandia. En segundo lugar, el estancamiento de los ingresos de los sectores medios o medios-bajos de los países ricos, es decir de amplios sectores de la clase obrera que se han visto perjudicados por la globalización. En tercer lugar, el autor recuerda que el principal beneficiado en las últimas décadas fue un minúsculo sector de la población mundial, el más rico, dando lugar a la emergencia de lo que llama "plutocracia global".

En su libro más reciente, Milanovic continúa este análisis y sostiene que en este período se consolidó en los países más desarrollados -si bien toma fundamentalmente a Estados Unidos como tipo ideal- lo que llama un "capitalismo liberal-meritocrático". 9 Una de sus características fundamentales fue el incremento de la desigualdad de ingresos, tanto por el estancamiento de los ingresos de la "clase media" y de la clase obrera como por un fuerte enriquecimiento de los sectores más ricos (la "plutocracia"). En sus análisis, este proceso se explica por una variedad de factores que, al igual que en el caso de la revolución industrial y la primera fase de la tradicional "curva de Kuznets", se dieron en el marco de un proceso de acelerado cambio tecnológico y globalización. En ambos libros, Milanovic introduce como elemento explicativo las relaciones (y los conflictos) entre las clases sociales, en la medida en que sostiene que la distribución del ingreso depende, en última instancia, del "poder relativo de negociación del capital y el trabajo". La globalización multiplicó la fuerza de trabajo disponible (para el capital), tanto por la integración de China y otros países comunistas al mercado mundial como por el crecimiento demográfico, lo cual debilitó la posición de los trabajadores; el crecimiento del "sector servicios", por su parte, también agravó la debilidad de los sindicatos.

Si bien Milanovic incorpora otros elementos (entre ellos las pautas matrimoniales del sector más rico de la población, una serie de políticas impositivas favorables a los millonarios, etc.), el punto central sigue siendo la

9 Branko Milanovic, Capitalism, Alone. The Future of the System that Rules the World (Cambridge, Harvard University Press, 2019), cap. 2. 
mayor disponibilidad de mano de obra y el debilitamiento de la posición negociadora de la clase obrera:

...es posible que el software de SAP, las computadoras de Lenovo y los iPhones de Apple hayan reemplazado los puestos de trabajo o reducido los salarios de las agencias de viajes, los empleados de hoteles, los contadores y los empleados de las tiendas, pero lo que podemos interpretar como un cambio tecnológico basado en las habilidades ocurrió porque el hardware barato para estos productos se produjo en países asiáticos con bajos salarios. ${ }^{10}$

\section{El “capitalismo político” y el lugar del comunismo en la historia}

Sea cual sea la opinión que nos formemos acerca de la creación de un nuevo tipo ideal para definir al capitalismo norteamericano contemporáneo, es indudable que resulta un acierto del autor su planteamiento de que no puede comprenderse sino en escala global, es decir tomando en consideración el papel que juega a nivel internacional el ascenso de China y otros países asiáticos. Impelido a buscar las causas históricas que llevaron al crecimiento económico de Asia, Milanovic apela a una explicación que, aunque resulta bastante obvia, le permite marcar una diferencia con buena parte de la literatura económica contemporánea: su argumento clave aquí es que el enorme crecimiento económico que muestran China y otros países de Asia en las últimas décadas es una consecuencia de las revoluciones comunistas del siglo XX y del modo en que, a partir de las décadas de 1970 y 1980, el gigante asiático avanzó hacia lo que denomina un "capitalismo político".

En Capitalism, Alone, Milanovic toma distancia de los análisis estadísticos y propone un peculiar ejercicio teórico e historiográfico acerca del "rol del comunismo en la historia", con un extenso capítulo y un apéndice que se alejan bastante de las estadísticas y se introducen en el campo historiográfico. $\mathrm{El}$ autor parte de una constatación evidente: durante el "siglo XX corto", como ya se dijo, la presión del movimiento obrero llevó al capital y los gobiernos de los países ricos a conceder reformas que en última instancia llevaron a una reducción de la desigualdad, pero en otras partes del mundo la característica fundamental del siglo pasado no fueron las concesiones de la burguesía sino el desarrollo y la victoria de procesos revolucionarios y de experiencias comunistas. El argumento central de Milanovic es que lo que llama "capitalismo político" (el alter-ego de ese "capitalismo liberal-

\footnotetext{
10 Milanovic, Global Inequality, 110.
} 
meritocrático" antes mencionado) es en la mayoría de los casos el producto de esas revoluciones comunistas en países coloniales.

Estas últimas, a su vez, habrían sido la consecuencia de una reformulación de la interpretación marxista, que según Milanovic -en una simplificación casi caricaturesca de la inmensa biblioteca teórica que ha abordado este puntosolo preveía para los países atrasados la repetición del camino de los más desarrollados. Por contraste, la "peculiaridad" del siglo XX, no contemplada según el autor en la interpretación marxista decimonónica, es que las revoluciones comunistas fueron en realidad la forma de salir del atraso y desarrollar el capitalismo en los países del "Tercer Mundo":

El comunismo es un régimen social que permitió que sociedades atrasadas

y coloniales abolieran el feudalismo, recuperaran independencia política y económica y construyeran un capitalismo doméstico (...) dicho de otro modo, fue un sistema de transición del feudalismo al capitalismo usado en sociedades menos desarrolladas y colonizadas. El comunismo es el equivalente funcional al ascenso de la burguesía en Occidente. ${ }^{11}$

Al mostrar con numerosos ejemplos que el capitalismo efectivamente no desarrollaba las sociedades atrasadas, sino que establecía vínculos de dependencia que perpetuaban el atraso, Milanovic recuerda que "la tarea de cualquier movimiento social en el Tercer Mundo era doble: transformar la economía doméstica modificando las relaciones de producción dominantes (...) y doblegar la dominación extranjera". Sostiene luego que "estas dos revoluciones - una revolución social cuyo objetivo último era el desarrollo y una revolución política cuyo objetivo último era la autodeterminación- se combinaron en una sola” ${ }^{12}$. Según Milanovic, China y Vietnam son los mejores ejemplos de revoluciones sociales y nacionales combinadas. Desafortunadamente, los lectores no encontrarán aquí ninguna referencia a los extensos debates que hubo acerca de este punto en el campo del marxismo y las teorías revolucionarias del siglo XX.

\section{El siglo XXI: el capitalismo en soledad}

Según Milanovic, desde la década de 1980, el mundo se caracteriza por el predominio absoluto del capitalismo. Reforzando la idea que da título a su último libro, señala que vivimos una época sin precedentes en la historia en cuanto a que todas las sociedades humanas están regidas por un mismo

11 Milanovic, Capitalism, Alone, 75-76.

12 Ibídem, 79. 
régimen social. Desde su punto de vista, "todo el planeta funciona según los mismos principios económicos: la producción organizada en función de la ganancia usando trabajo asalariado legalmente libre y mayormente capital privado, con coordinación descentralizada". ${ }^{13}$

Como ya señalamos, este dominio global del capitalismo, de acuerdo a la perspectiva del autor, expresa en realidad, la coexistencia de dos "tipos" de capitalismo: el "liberal meritocrático", que predomina en el "mundo occidental", y el "político", cuyo principal exponente es China y es consecuencia de una revolución comunista. Según Milanovic, este mundo menos desigual es también más equilibrado en términos geográficos y políticos: a través de sus obras, subyace la impresión de una tendencia hacia la convergencia que licúa la idea de imperialismo como herramienta analítica y retorna -esta vez a nivel global- a posiciones similares a las de Kuznets.

Respecto al capitalismo "liberal-meritocrático", Milanovic propone compararlo con otros dos tipos que habrían existido en el pasado: el capitalismo "clásico" (cuyo modelo sería el Reino Unido hasta 1914) y el capitalismo "socialdemócrata" del siglo XX (cuyo modelo serían Europa y Estados Unidos entre 1945 y la década de 1980). Varias particularidades del capitalismo actual, según Milanovic, son similares a las del capitalismo "clásico": hay un aumento de la participación del capital en el ingreso total (es decir el capital se queda con una parte mayor de la torta respecto al trabajo), los ricos tienden a casarse y formar familias entre sí, hay una alta correlación entre los ingresos de los padres y los hijos, etc. Hay una novedad significativa, sin embargo, y es que las personas que poseen un ingreso muy elevado por su capital también reciben un ingreso elevado por su trabajo (profesionales, ejecutivos, etc.).

Mientras que las personas que encabezaban la distribución de ingresos en el capitalismo clásico eran financistas, rentistas y propietarios de grandes holdings industriales (que no son contratados por nadie y, por lo tanto, no tienen ingresos laborales), hoy en día un porcentaje significativo de las personas que encabezan la distribución de ingresos son gerentes altamente remunerados, diseñadores de páginas web, médicos, banqueros de inversión y otros profesionales de élite. ${ }^{14}$

¿Cuáles son las características principales del "capitalismo político" en la actualidad? Según Milanovic, sus tres rasgos fundamentales son la presencia de "una burocracia extremadamente eficiente e inteligente en términos

13 Ibídem, 2.

14 Ibídem, 17. 
tecnocráticos" a cargo del sistema, la ausencia de un "estado de derecho" ("rule of law”) y la capacidad del Estado para defender los intereses nacionales y al mismo tiempo controlar al sector privado. ${ }^{15}$ Milanovic también destaca dos contradicciones: en primer lugar, el hecho de que esta burocracia tiene que ser muy eficiente al tiempo que funciona sin un claro marco legal; en segundo, que el sistema promueve la corrupción (que aumenta las desigualdades) y al mismo tiempo debe reducir la desigualdad para no perder legitimidad. El sistema funciona siempre en un equilibrio precario, sin que pueda actuar plenamente el imperio de la ley pero también evitando que se descontrole la corrupción. ${ }^{16}$

${ }_{¿}$ Es China capitalista? Milanovic responde afirmativamente, planteando que cumple con los tres criterios de la “definición estándar de Marx y Weber”, a saber: la mayor parte de la producción se lleva adelante usando medios de producción en manos privadas, la mayoría de los trabajadores son asalariados y la mayoría de las decisiones sobre producción y precios se toman de manera descentralizada. En este punto, el autor aporta datos valiosos acerca de las relaciones sociales de producción en la historia reciente del país asiático: en 1978, prácticamente el $100 \%$ de la producción industrial del país era producida por empresas estatales; en 1998, esa cifra se había reducido al 50\% y en 2018 llegaba a apenas 20\%. Según Milanovic, la tendencia es aún más marcada en el ámbito agrícola. A lo largo de sus textos, el autor admite la complejidad que implica analizar las relaciones de propiedad en China, dada la peculiar imbricación de propiedad privada, extranjera, comunal, provincial, etc. Sostiene, sin embargo, que en términos generales el peso de las empresas estatales en la producción y el empleo es similar al de, por ejemplo, Francia en la década de 1980.

Milanovic avanza algunos análisis en el terreno de la estructura de clases de China y la emergencia lenta pero sostenida de una "clase empresarial" surgida en los últimos años, porque no tiene vínculos con la burguesía prerrevolucionaria. El rasgo característico, en su opinión, es que "los capitalistas se enriquecen sin ejercer el poder político”. ${ }^{17}$ Esta autonomía de la burocracia del Estado respecto a la clase burguesa se convierte por lo tanto en el elemento decisivo: según Milanovic, "la opacidad de distintas formas de

15 Ibídem, 91.

16 ¿Qué países entran en esta descripción? Milanovic enumera once: China, Vietnam, Malasia, Laos, Singapur, Argelia, Tanzania, Angola, Botsuana, Etiopía y Ruanda. En conjunto, suman un cuarto de la población mundial y un quinto de su producción y crecen de forma muy acelerada: en 1990 constituían el 24\% de la población mundial pero solo el 5,5\% de su producción.

17 Milanovic, Capitalism, Alone, 106. 
propiedad no es un 'error', ni algo transitorio que necesita ser 'corregido', sino la condición básica de existencia del capitalismo político". ${ }^{18}$ Hay precisamente un paralelo entre esta hibridez en las formas de propiedad y el hecho de que no rige plenamente el "estado de derecho". En conclusión, en China "el gobierno contribuye a los intereses de la burguesía, pero siempre y cuando estos intereses no vayan en contra de los objetivos del stado (es decir, de la elite que controla el estado)". ${ }^{19}$

\section{Conclusión}

Los estudios sobre desigualdad económica están ocupando un lugar central en debates actuales, tanto en el terreno de la economía como en distintas disciplinas sociales. En este contexto, los trabajos recientes de Milanovic tienen, en mi opinión, dos méritos: por un lado, sistematizan una cantidad de información estadística y la ponen al alcance de un público lector más amplio; por el otro, buscan poner la discusión sobre la desigualdad de ingresos en una clave histórica, de larga duración y que presta especial atención a la forma en que se estructuran las relaciones laborales y los conflictos de clase en las diferentes sociedades. Milanovic, por otra parte, no es historiador ni ha realizado investigaciones en clave histórica: en ese sentido muchas de las reflexiones que propone en sus libros resultarán extremadamente simplistas para un lector especializado en la literatura histórica, y particularmente en la historia del trabajo, y muestran que en buena medida el público que tiene en mente el autor es otro.

A pesar de esto último -en realidad debido a esto último- los temas que abordan los libros recientes de Branko Milanovic deben interesar seriamente a los estudiosos de la historia de los trabajadores y trabajadoras, así como estimular sus aportes. En lo fundamental, esto se debe a que hay un eje que atraviesa todo el análisis: aunque parezca una obviedad, la cuestión de la desigualdad económica no puede analizarse sin prestar atención a las relaciones laborales y a las relaciones entre las clases, y eso solo puede hacerse en perspectiva histórica. Los diversos ciclos de ascenso y descenso de la desigualdad de ingresos en las épocas preindustriales -sin importar cómo se midan e incluso si se pone en discusión la posibilidad misma de medirlos debido a la escasez de fuentes- solo pueden comprenderse prestando atención a la dinámica del conflicto social, lo cual incluye desde las tensiones relativas a la distribución de la renta hasta los conflictos bélicos. Milanovic repone

18 Ibídem, 116.

19 Ibídem. 
algunas de estas cuestiones en sus libros, que por otra parte han sido largamente estudiadas en una biblioteca inmensa que también puso en primer plano la importancia del trabajo no remunerado indispensable para garantizar la reproducción social que realizan fundamentalmente las trabajadoras mujeres, un tema pasado por alto en los volúmenes aquí reseñados. Analizar el "gran despegue" tecnológico y productivo de la Revolución Industrial, por su parte, supone prestar atención a las transformaciones en las relaciones de clase que implicó el desarrollo capitalista, y el indiscutible incremento de la desigualdad económica que trajo como consecuencia -algo aceptado incluso en una perspectiva "optimista" como la de Kuznets-. Se trata de temas que fueron y siguen siendo la base de una enorme literatura en la mejor tradición de la historia social, que debe poner su voz en estos debates.

Como bien puso de relieve Piketty, la reducción de la desigualdad en los países ricos durante el "siglo XX corto", por su lado, es imposible de comprender sin analizar las relaciones de clase, la organización política del movimiento obrero, etc. Las limitaciones de la "curva de Kuznets” y sus variantes de "teoría del derrame" son demasiado evidentes, y aunque Milanovic parece interesado en salvar todo lo posible la idea de fondo de una convergencia, agregando la noción de "ciclo", lo cierto es que buena parte de sus análisis contribuyen a asestarle una nueva crítica: para comprender la economía del siglo XX hay que examinar su política, y para comprender su política hay que poner en primer plano los conflictos entre el capital y el trabajo y la historia de la organización obrera. A su vez, las tendencias más recientes (reducción de la desigualdad a nivel global por la mejora del ingreso de la "clase media global", aumento de la desigualdad en Europa y América del Norte) solo pueden explicarse si analizamos el aumento en la mano de obra disponible debido a la globalización y los cambios en las relaciones de clase que provoca. En suma, el "hilo rojo" para comprender la historia contemporánea y, como parte de ella, las oscilaciones de la desigualdad económica, es el modo en que se estructura la producción de la vida material (las relaciones sociales de producción) y el modo en que se organiza la relación entre las clases, a nivel global. 